Hojas y Hablas No. 18, julio-diciembre de 2019. ISSN en línea 2539-3375

\title{
Responsabilidad Social Empresarial competitiva en Mipymes de la Sabana de Occidente, Cundinamarca
}

\author{
Competitive Corporate Social Responsibility in MSMEs of Sabana de \\ Occidente, Cundinamarca
}

\author{
Francisco Javier Zipasuca Quemba \\ Fabio Orlando Cruz Páez ${ }^{2}$ \\ Juan de Jesús Rojas Ramírez ${ }^{3}$ \\ Omar Fernando Muñoz ${ }^{4}$ \\ DOI: 10.29151/hojasyhablas.n18a1
}

\section{Resumen}

El presente artículo de investigación muestra la importancia de la Responsabilidad Social Empresarial (RSE) y la incorporación de la misma en la planeación de las Mipymes de la Sabana de Occidente en Cundinamarca, Colombia. La metodología fue de corte mixto con diseño transversal, mediante una encuesta dirigida a los gerentes de 206 pequeñas empresas, 93 de medianas empresas y 771 de microempresas, ubicadas en ocho municipios del departamento de Cundinamarca. En el análisis se exploran las variables teóricas de Responsabilidad Social Empresarial, competitividad, stakeholders, pymes y medio ambiente, evidenciando que para implementar la RSE en Mipymes es fundamental aumentar la comprensión de este concepto y fortalecer la planeación. Adicionalmente, se comprobó que el mejor punto de partida es trabajar la relación con los stakeholders, que están más conectados con el núcleo del negocio, así como las relaciones comunitarias y la sensibilización ambiental.

Palabras clave: Responsabilidad Social Empresarial; competitividad; stakeholders; pymes; medio ambiente.
This research paper shows the importance of Corporate Social Responsibility (CSR) and the incorporation of it in the planning process of micro, small and medium-sized enterprises (MSMEs) in Sabana de Occidente, province of Cundinamarca, Colombia. The investigation used a mixed-type methodology with a cross-sectional design, applying a survey to managers of 206 small companies, 93 medium-sized companies and 771 micro-enterprises, all of them located in eight municipalities of Cundinamarca. The analysis explored from theoretical variables of Corporate Social Responsibility, competitiveness, community relations, stakeholders and the environment, evidencing that to implement a CSR perspective in MSMEs requires to understand this concept and strengthen the planning process, firstly. In addition, the best starting point is to work on the relationship with stakeholders that are more connected to the core of the business, as well as community relations and environmental.

Keywords: Corporate Social Responsibility; competitiveness; stakeholders; SMes; environment.

\footnotetext{
${ }^{1}$ Magíster en Ciencias de la Educación. Especialista en Pedagogía y Docencia Universitaria. Teólogo. Licenciado en Filosofía y Ciencias Religiosas. Docente Universidad de Cundinamarca, Colombia. E-mail: fjzipasuca@ucundinamarca.edu.co ORCID: 0000-0003-1599-9649

${ }^{2}$ Magíster en Administración de Organizaciones. Docente Universidad de Cundinamarca, Tutor Universidad Santo Tomás, Instructor SENA del Centro de Biotecnología Agropecuaria, Colombia. E-mail: focruz@ucundinamarca.edu.co ORCID 0000-0001-7834-2762

${ }^{3}$ Magíster en Dirección Estratégica con énfasis en tecnologías de la información. Administrador de Empresas, Especialista en Docencia Universitaria, Coordinador Universidad de Cundinamarca, Colombia. E-mail: jrojas@ucundinamarca.edu.co ORCID: 0000-0001-7323-4180

${ }^{4}$ Magíster en Gestión de Calidad para la Educación Superior Universidad, Especialista en Docencia Universitaria, Administrador de Empresas, Coordinador Universidad de Cundinamarca. E-mail: omarf@ucundinamarca.edu.co ORCID: 0000-0003-4368-9904
} 


\section{Introducción}

En la Sabana de Occidente se refleja, al igual que en Colombia, que "la capacidad emprendedora da gran importancia a la competitividad de las empresas que se crean no solo por crearlas sino como una oportunidad de negocio" (Cruz, 2015: 102), es así como la cantidad de unidades productivas formalizadas siguen creciendo en la respectiva provincia y genera mayor competitividad en Mipymes cuando apropian la Responsabilidad Social Empresarial (en adelante RSE), como uno de los ejes de interacción en los modelos organizacionales.

La Responsabilidad Corporativa es el compromiso continuo por parte de las empresas para comportarse de una manera ética y contribuir al desarrollo económico sostenible, al mismo tiempo que se mejora la calidad de vida de los trabajadores y sus familias, así como de la comunidad local y de la sociedad en general (World Business Council for Sustainable Development [WBCSD] citado en Corporación Universitaria Minuto de Dios, 2018: 1).

Por ello, la importancia de la RSE en Mipymes con el fin de ser competitivos en el mercado nacional e internacional se debe promover desde la apropiación y empoderamiento de las empresas puesto que,

El concepto de RSE debe enriquecerse con estrategias que den la oportunidad de generar valor económico, social y ambiental al integrar a las Mipymes en la cadena de valor como socios de negocio. ¿Cómo?, invirtiendo en su desarrollo, acercándoles herramientas financieras y tecnológicas e insertándolas eficientemente en los mercados. En pocas palabras: aumentando su productividad. (La Republica.net, 2016: 1).

La Cámara de Comercio de Facatativá (2018) afirma que "la región Sabana de Occidente continúa su liderazgo como la provincia líder en crecimiento de unidades formalizadas y renova- das, concentrando el $66 \%$ del total de la base empresarial de las seis provincias de la jurisdicción de la CCF” (p. 5). Es por ello, que se debe promover la responsabilidad social empresarial desde la provincia, en pro del desarrollo competitivo en Cundinamarca para la sociedad. Así mismo, el mismo ente establece que:

En la región Sabana de Occidente se localiza el mayor número de empresas (62\%) seguida por Gualiva (20\%). Al igual que el resto del país, la estructura empresarial formalizada que más predomina en la jurisdicción de la CCF es la microempresa $(98,4 \%)$, las pymes participan con cerca del 1,3\% y la gran empresa representa el $0,3 \%$, no obstante, se mantiene la tendencia de las grandes firmas a localizarse en la provincia de Sabana Occidente, como resultado del desarrollo de esta provincia las medianas y grandes empresas se localizan en la Sabana, teniendo en cuenta la distribución de tamaño de empresa según la Ley 905 de 2004 (Cámara de Comercio de Facatativá, 2018: 84).

Igualmente, se evidencia que:

Para muchas pequeñas y medianas empresas (PYMES) la Responsabilidad Social Empresarial (RSE) es un tema desconocido, lejano o exclusivo de los grandes corporativos. Sin embargo, las PYMES también pueden involucrarse en el tema; la diferencia es simplemente el alcance y la escala de los programas y actividades a implementarse (Instituto Nacional de Contadores Públicos, 2016: 1).

De ahí que los estudios señalen el escaso número de Mipymes que implementan prácticas de RSE. Esta situación no es extraña, si se considera que las herramientas de referencia para emprender una gestión más responsable están basadas en la experiencia de multinacionales y empresas grandes, sin tomar en cuenta las características y necesidades particulares de las Mipymes. Por ejemplo, la Guía para Pymes de Global Reporting Iniciative (GRI), las guías de la Corporación Fi- 
nanciera Internacional (CFI) y la guía internacional en responsabilidad social ISO 26000, contienen modelos de lo que debería hacerse, así como recomendaciones frente a aspectos técnicos que son entendidas por las Mipymes como exigencias difíciles de cumplir de cara a sus urgencias del día a día y a la disponibilidad limitada de recursos. Por otra parte, las Mipymes están lejos de ser un grupo homogéneo, y sus características y necesidades son variadas.

La presente investigación se llevó a cabo en tres fases durante el último semestre del 2015 y todo el año 2016. La pregunta de investigación buscó constatar si la RSE era un factor importante dentro de la planeación, directamente relacionada a la competitividad para 1.070 Mipymes activas en los ocho municipios que componen la Sabana de Occidente. Del mismo modo, se identificó el tipo de acciones de RSE que estas empresas ya están implementando en el entorno de la competitividad empresarial.

La literatura relevante indicó que la RSE puede ser implementada en las Mipymes si la dinámica de la gestión empresarial, la capacidad gerencial y la cultura organizacional favorecen el cambio, la toma de decisiones, la medición de resultados y la mejora continua. En este sentido, la existencia de una estrategia organizacional, de una visión y una misión, de objetivos claros, de canales de comunicación claros entre la gerencia y los empleados, y de una cultura de medición, son factores cruciales para determinar si se está avanzando o si se está preparada para avanzar hacia una gestión más responsable y competitiva.

De esta manera, se formuló la siguiente hipótesis: la aplicación de estrategias de desarrollo organizacional desde la Responsabilidad Social Empresarial en las micro, pequeñas y medianas empresas (Mipymes), fundamentada desde la apropiación y fortalecimiento de factores organizacionales en modelos organizacionales, la relación con los stakeholders y el cuidado del medio ambiente, pueden potencializar la competitividad de las Mipymes en la Sabana de Occidente, tanto para la sociedad, la empresa, el Estado y la Universidad en la interacción de las mismas, irradiando en el mercado local y regional.

La pregunta problema de la investigación se dirigió a ¿Cómo influye la gestión de la Responsabilidad Social Empresarial en la competitividad organizacional desde la planeación en las Mipymes de la provincia Sabana de Occidente de Cundinamarca? A su vez, el nivel de sensibilidad que la micro, pequeña o mediana empresa tenga hacia los factores externos que la afectan, y hacia el relacionamiento e identificación de necesidades de sus colaboradores, clientes y otros stakeholders como proveedores, distribuidores y acreedores, la hacen estar más preparada para el cambio. Para este efecto, los representantes legales de estas empresas respondieron a una encuesta que apuntaba a la exploración de los siguientes puntos: 1) aspectos organizacionales; 2) la relación de las empresas con los stakeholders; y 3) la relación de las empresas con el entorno y el cuidado del medio ambiente.

Los hallazgos de la investigación permitieron afirmar que la gestión de la RSE de las pequeñas empresas de esta región es aún débil y debe fortalecerse. Si bien se identificaron algunas acciones de RSE dentro de las empresas estudiadas, es posible decir que estas acciones no son el resultado de una comprensión exhaustiva del concepto de RSE, ni de una integración sólida del mismo en la planeación y en la gestión operativa de las empresas para ser más competitivas en el actual contexto global, frente a los modelos organizacionales que inciden en el desarrollo socioeconómico, cultural y ambiental de la comunidad.

\section{Metodología}

La metodología empleada por la investigación contó con un diseño mixto, el cual hace "uso de estrategias cuantitativas y cualitativas" (Guerreo, Lenise y Ojeda, 2016: 248). En el caso de lo 
cualitativo, se consultaron bases de datos para la búsqueda de antecedentes sobre las Mipymes y la responsabilidad social de las empresas. Y para el carácter cuantitativo, se elaboró una encuesta a escala Likert sobre la responsabilidad social de las Mipymes en las empresas de la Sabana de Occidente, en ocho municipios: Bojacá, El Rosal, Facatativá, Funza, Madrid, Mosquera, Subachoque y Zipacón.

De tal forma que, para la recolección de la información se aplicó una encuesta a los representantes legales de un total de 1.070 Mipymes de la región, distribuidas en 206 pequeñas empresas, 93 de medianas empresas y 771 de microempresas. El objetivo de la encuesta fue ubicar el nivel de inclusión de los siguientes tres grupos de aspectos dentro de la planeación organizacional de cada empresa:

- Factores organizacionales: se refieren al nivel de conocimiento del concepto de RSE, a la existencia de objetivos de RSE y a la asignación de una dependencia de RSE; a la existencia de una misión y una visión explícitas orientadas al tema, con valores y principios organizacionales definidos; así como a la aplicación de herramientas de planeación, y a la fijación de objetivos y medición de resultados.

- Relación con los stakeholders: analiza la sensibilidad de la empresa frente a las tendencias y cambios de su entorno, así como a las necesidades de sus clientes y otros grupos de interés importantes como colaboradores, proveedores, distribuidores y acreedores.

- Cuidado del medio ambiente y del entorno: indaga por la existencia de capacitación en materia de cuidado ambiental al interior de la organización y a la promoción del trabajo voluntario de los empleados con la comunidad.
Para la recolección de la información se contactó a los representantes legales de las empresas personalmente, por vía telefónica y por correo electrónico. Los datos fueron procesados estadísticamente. En primer lugar, la información se separó de acuerdo al tamaño de las empresas: una pequeña empresa cuenta con una planta de personal entre 11 y 50 empleados y con unos activos totales entre 501 y 5000 Salarios Mínimos Mensuales Legales Vigentes (SMMLV); una empresa mediana cuenta con una planta de personal entre 51 y 200 empleados, y con unos activos totales entre $5001 \mathrm{y}$ 30.000 SMMLV; y una microempresa tiene entre 1 y 10 empleados, y activos totales inferiores a 500 SMMLV, excluida la vivienda.

Durante la Fase 1 de la investigación, se encuestaron 206 pequeñas empresas correspondientes al $48 \%$ de la totalidad de pequeñas empresas en la zona. Para la Fase 2, se encuestaron 93 medianas empresas, correspondientes al 100\% de empresas de este tipo en la zona; y en la Fase 3 se encuestaron 771 microempresas, correspondientes al 100\% de microempresas activas en la provincia de Sabana de Occidente del departamento de Cundinamarca.

\section{Resultados y discusión}

A continuación, se aprecian los resultados y discusión de los tres aspectos o variables estratégicas en las que se agrupó y sistematizó la información en torno a la Responsabilidad Social Empresarial.

\section{Factores organizacionales}

Los resultados de la encuesta permitieron sustentar que las pequeñas empresas de la Sabana de Occidente deben fortalecer la gestión de RSE en diferentes ámbitos. Un primer hallazgo fue que más de la mitad de las empresas manifestaron no 
conocer qué es la RSE, o conocerlo tan solo parcialmente; esta tendencia fue constante para los tres tipos de empresa (54\% pequeñas, 53\% medianas y 57\% microempresas). En contraste, sólo un $46 \%$ de las pequeñas empresas, un $47 \%$ de las medianas, y un $43 \%$ de las microempresas, reportaron estar al día con el concepto y utilizarlo en algún ámbito para el funcionamiento corporativo.

Surgen entonces dos preguntas a saber: la primera es ¿con qué relacionan el concepto de RSE las empresas?, y la segunda es ¿cómo aplican en la práctica los conceptos que conocen? Cuando se les preguntó si aplicaban el concepto directamente en la empresa, sólo el $28 \%$ de las pequeñas empresas, el 24\% de las medianas, y el 5\% de las microempresas reportaron hacerlo siempre. En contraste, el $72 \%$ de las pequeñas, el $76 \%$ de las medianas, y el 95\% de las micro reportaron no hacerlo nunca, o muy rara vez. Es evidente que el porcentaje de empresas que aún no aplican la RSE es alto, en especial en el caso de las microempresas.

Es notable también que, en su mayoría, todos los tipos de empresas encuestadas relacionan el concepto de RSE con el cuidado del medio ambiente y del entorno, seguido por ayudar a la comunidad y mejorar la calidad de vida laboral así: $28 \%$ pequeñas empresas, $24 \%$ las medianas y un $5 \%$ las microempresas. En contraste, para los tres tipos de empresa, el factor "disminución de impuestos" no es tan relevante cuando de RSE se trata. Por otro lado, se observó que el factor reputacional tiene más peso para las medianas empresas. Un 18\% de estas empresas reportaron relacionar el concepto de RSE con un mejoramiento en su imagen, frente a un 3\% de las pequeñas y a un $8 \%$ de las microempresas.

Para integrar sólidamente la RSE en la planeación y en la gestión de un negocio, es necesario que las empresas cuenten con unos valores y principios organizacionales claros, que sean socializados y cumplidos en la operación. Este aspecto es cumplido satisfactoriamente por más de la mitad de las pequeñas y medianas empresas (58\% y 56\% respectivamente). Las medianas empresas muestran un avance superior a las pequeñas empresas, dado que un $18 \%$ de estas últimas reportó no tener establecidos dichos principios en absoluto, mientras que un $12 \%$ de las medianas empresas reportó tenerlos parcialmente establecidos. En el caso de las microempresas, el panorama es diferente, el $37 \%$ no cuenta con principios y valores, o cuenta con ellos parcialmente, y menos de la mitad (46\%) los socializa y cumple en la práctica, evidenciando la necesidad de diseño, establecimiento y gestión en las Mipymes en la provincia de Sabana de Occidente.

Además de los valores organizacionales, es también importante que las empresas tengan definidas una visión y una misión claras, en las que puedan incluir la RSE como un factor orientador. En este sentido se encontró que un 75\% de las pequeñas empresas tienen una misión y una visión bien definidas, mientras que el 25\% no las han establecido nunca. Sorpresivamente, menos de la mitad $(47 \%)$ de las medianas empresas tiene establecida su misión y visión, y un $28 \%$ no lo han hecho nunca y el 25\% lo han intentado establecer. En el caso de las microempresas, el 12\% cuentan con una misión y una visión, mientras que el 50\% no lo ha hecho nunca y el $48 \%$ lo han intentado realizar. 


\section{Gráfica 1. Misión - Visión en la Responsabilidad Social Empresarial}

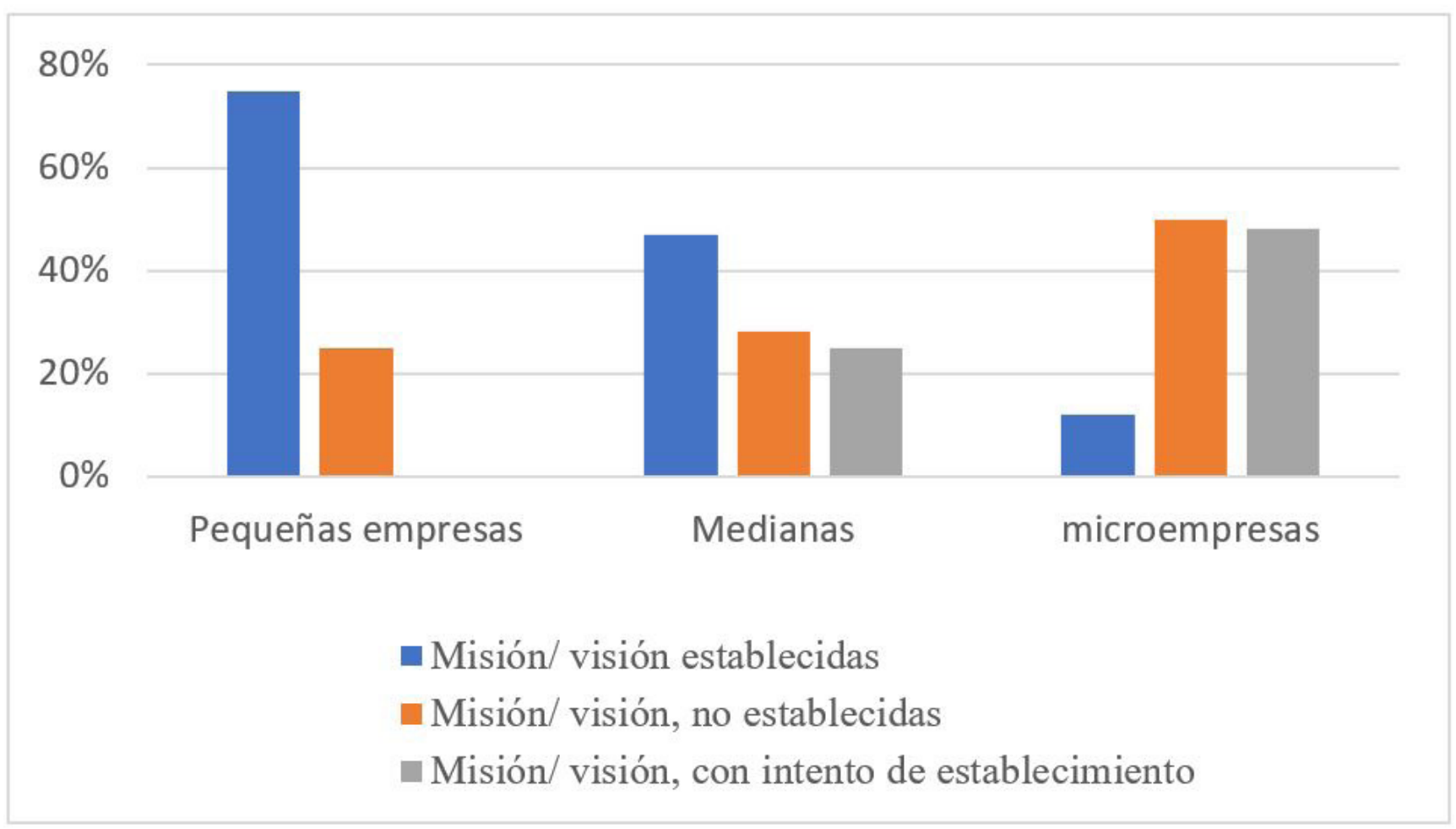

Fuente: Elaboración propia, 2019.

En lo que se refiere a la importancia de la planeación estratégica para las Mipymes de la Sabana de Occidente, se encontró que un porcentaje relativamente alto de las pequeñas y medianas empresas (69\% y $77 \%$ respectivamente) aplican siempre o de manera regular herramientas de planeación organizacional, que influyen significativamente en sus decisiones. En el caso de las microempresas, este número se reduce a un poco menos de la mitad (44\%). Las herramientas de planeación organizacional favorecen significativamente la disposición hacia el cambio y la aplicabilidad de nuevas medidas; para que una gestión de RSE sea sólida, y permee realmente los diferentes ámbitos de la organización, se requiere de un proceso de planeación estructurado y sistemático. El alto porcentaje de Mipymes que no han introducido estas herramientas es una señal de alerta clara.

Existe otra señal que indica preparación para el cambio hacia una gestión más sostenible, relacionada con la disciplina para realizar periódicamente evaluaciones de resultado. Una evaluación de resultados, realizada siempre o algunas veces, se evidencia en un $74 \%$ de las pequeñas empresas, un $61 \%$ de las medianas y un $62 \%$ de las microempresas.

Si dentro de los valores organizacionales de misión y visión de las empresas está incluida la RSE, ésta debería estar definida dentro de la planeación de la organización, lo cual infiere en la competitividad organizacional a nivel de las pymes en la provincia de la Sabana de Occidente. $\mathrm{Al}$ explorar este punto, se encontró que el $46 \%$ de las pequeñas empresas dice incluir la RSE dentro de su planeación estratégica, mientras que el 25\% lo hace ocasionalmente, y el $29 \%$ nunca. En el caso de las medianas empresas, el $38 \%$ incluye siempre la RSE en su planeación, mientras que el 35\% ocasionalmente y el 27\% nunca. En el caso de las microempresas, sólo un 6\% incluye siempre la RSE 
en su planeación, el 37\% algunas veces y el 57\% nunca. Esto quiere decir que las pequeñas y medianas empresas en las que se socializan y se ponen en práctica valores y principios organizacionales, no siempre se habla de principios y valores directamente asociados con la RSE. En el caso de las microempresas es evidente que el concepto no ha logrado llegar a la planeación de la empresa.

\section{Gráfica 2. Inclusión de la Responsabilidad Social Empresarial en la planeación corporativa}

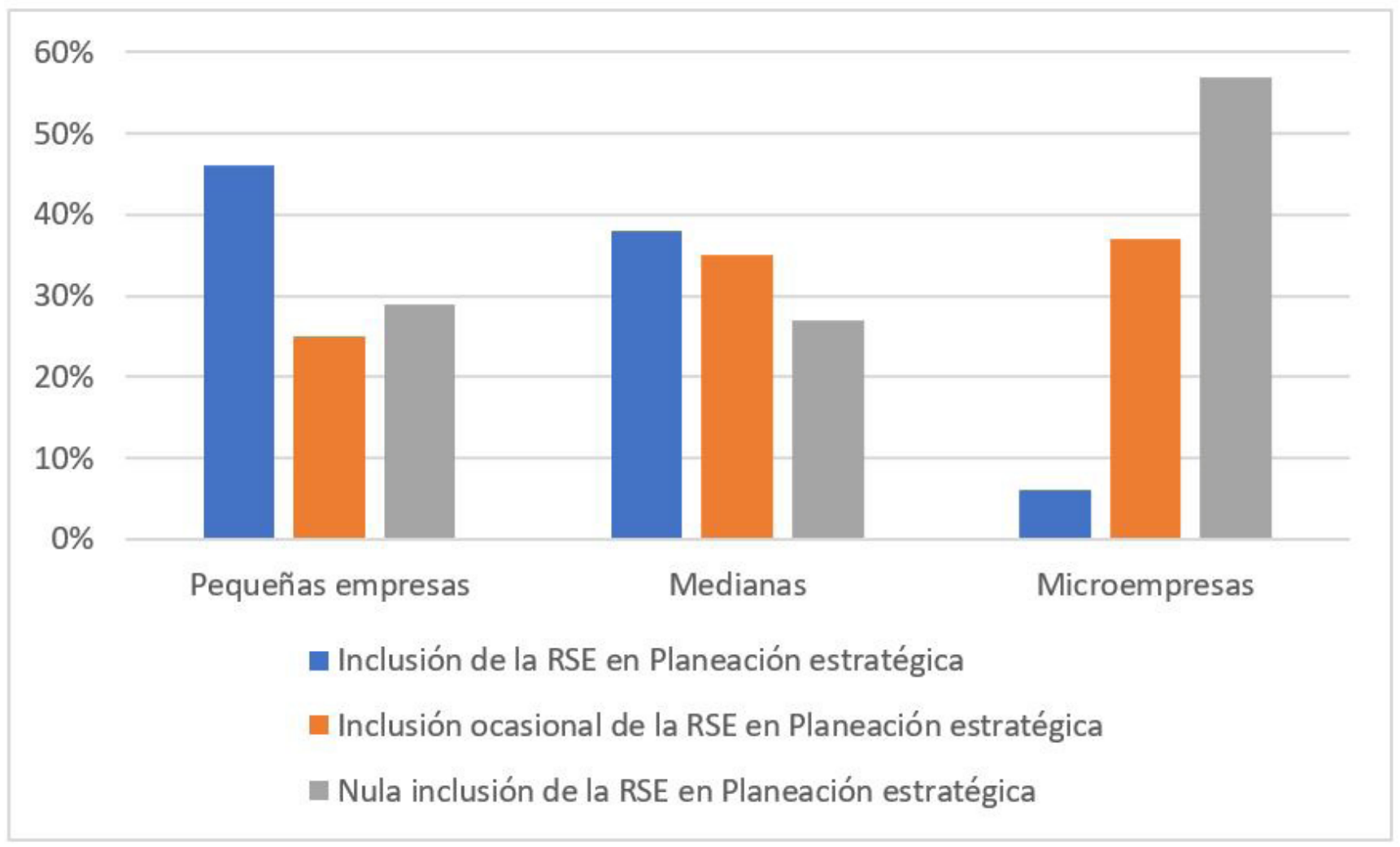

Fuente: Elaboración propia, 2019.

Por otra parte, para que la aplicación de la RSE en la operación de la empresa sea más efectiva, es bueno tener una dependencia especial o al menos una persona que reciba la responsabilidad directa de ejecutar las tareas de RSE. En este sentido, sólo el $26 \%$ de las pequeñas empresas, el $19 \%$ de las medianas, y el $2 \%$ de las micro, manifestaron contar con una dependencia o persona orientada específicamente a la RSE. En contraste, más de la mitad de los tres tipos de empresa (51\%, $73 \%$ y $72 \%$ respectivamente) reportaron no contar nunca con una dependencia o persona a cargo de la RSE, y el 23\%, $8 \%$ y el $26 \%$ lo hacen empoderando una persona de la empresa rara vez.

\section{Relación con los Stakeholders}

Un aspecto fundamental de la RSE es gestionar adecuadamente la relación de la empresa, tanto con los stakeholders externos como internos, frente a la competitividad empresarial. Para lograrlo, es importante que las empresas sean sensibles a lo que ocurre en su entorno y a las necesidades de los grupos de interés con el fin de tomar decisiones en relación a ellos.

En este sentido, se encontró que las Mipymes de la Sabana de Occidente son sensibles a los cambios del entorno y a las necesidades de los stakeholders externos, tales como clientes, proveedores, 
distribuidores y acreedores. El 73\% de las pequeñas empresas, el $67 \%$ de las medianas y un $64 \%$ de las microempresas observa y prevé con regularidad los cambios económicos, sociales, políticos, legales y tecnológicos del entorno. Si bien es aún alto el porcentaje de Mipymes que aún no lo hacen, o lo hacen muy rara vez, la mayoría de las empresas muestran esta disposición para leer el entorno y prepararse frente a los cambios.

Para los tres tipos de empresas, el cliente es el stakeholder más importante, pues de este depende en mayor medida su sostenibilidad económica. El $90 \%$ de las pequeñas empresas, el $86 \%$ de las medianas y el $74 \%$ de las microempresas, analizan siempre o muy regularmente los cambios y las necesidades de los clientes en el actual mercado competitivo. En cuanto a los proveedores, distribuidores y acreedores, la sensibilidad de las Mipymes también es alta. El $80 \%$ de las pequeñas empresas, $81 \%$ de las medianas y $51 \%$ de las microempresas, siempre o muy regularmente analizan las necesidades de estos grupos de interés.

Si bien esta sensibilidad frente al entorno habla de la dependencia que las Mipymes tienen frente a los grupos interés externos para garantizar la sostenibilidad económica, esto puede constituirse como un factor organizacional positivo que las prepara para introducir, en el futuro los objetivos de las RSE en el manejo de dichas relaciones. En este sentido, es importante aclarar que, si bien estas empresas leen las necesidades de sus stakeholders externos y reaccionan a ellas, no necesariamente lo están haciendo con un enfoque de RSE. Por otra parte, los colaboradores son, sin duda, un stakeholder importante para las Mipymes. La mayoría de las pequeñas, medianas y microempresas (91\%, el 74\% y $60 \%$,

En coherencia con lo anterior, las pequeñas, medianas y microempresas han desarrollado mecanismos para atender bien a los clientes de los diferentes segmentos del mercado que tienen las Mipymes en la Sabana de Occidente, (90\%, 76\% y
$80 \%$ respectivamente). Del mismo modo, más de la mitad de ellas les ofrecen beneficios extralegales (64\%, 68\% y $60 \%$ respectivamente). La capacitación y las campañas de promoción del desarrollo son también factores importantes para más de la mitad de las pequeñas y medianas empresas $(75 \%$ y $61 \%$ respectivamente), las cuales ofrecen siempre o con regularidad estos beneficios. En el caso de las microempresas, este factor está aún por desarrollarse con más fuerza, pues solo un $46 \%$ ofrecen capacitaciones y promueven el desarrollo entre los colaboradores frecuentemente.

La promoción y la defensa de los derechos humanos dentro de la organización son factores fundamentales para garantizar el bienestar de los colaboradores. La encuesta exploró la sanción a la discriminación en el entorno de trabajo, como un indicador de preocupación y diligencia cuando se presentan irregularidades. En este sentido se encontró que el 58\% de las pequeñas empresas, el 78\% de las medianas y el 51\% de las microempresas, sancionan siempre, o algunas veces, las actitudes de discriminación dentro de la organización. Esto significa que la mayoría de los tres tipos de empresa son sensibles con el tema y toman medidas al respecto, aunque se aprecia igualmente un porcentaje alto de ellas que no lo hacen: $42 \%$ de pequeñas, $22 \%$ de medianas y $49 \%$ de microempresas.

Entonces, la relación de las Mipymes con los colaboradores, clientes, proveedores, acreedores y distribuidores es, en su mayoría, estrecha y sensible; por ello se constituye como un campo en el cual la introducción de objetivos y planes de RSE podría obtener muy buenos resultados relativamente rápido.

\section{Cuidado del medio ambiente y del entorno}

A pesar de lo que se evidenció frente a lo mencionado en la primera sección de este análisis, la mayoría de las empresas relacionan el concepto de RSE con el cuidado del medio ambiente y del 
entorno, y se aprecia que este aspecto está poco desarrollado en los tres tipos de empresas. Para el caso de las pequeñas empresas, sólo el 15\% involucra a la comunidad para las discusiones que atañen la sostenibilidad social, económica y ambiental de la empresa, y el 23\% dice hacerlo algunas veces. En el caso de las medianas empresas, únicamente el $4 \%$ dice hacerlo siempre y el $12 \%$ algunas veces. Finalmente, el $2 \%$ de las microempresas reportan hacerlo siempre y un $31 \%$ algunas veces. Estos resultados indican que es necesario despertar conciencia entre las Mipymes frente a la importancia que tienen otros stakeholders externos, y su influencia en la sostenibilidad social y ambiental del negocio, debido al muy alto porcentaje de nulo cuidado al respecto: $62 \%, 84 \%$ y $67 \%$.

Por otra parte, se encontró que las capacitaciones en materia ambiental son un aspecto que también podría desarrollarse con mayor frecuencia y que ayudaría a incrementar la competitividad de las empresas en el contexto globalizado desde la provincia de Sabana de Occidente. El 29\% de las pequeñas

\section{Gráfica 3. Cuidado del medio ambiente por Mipymes de la Sabana de Occidente}

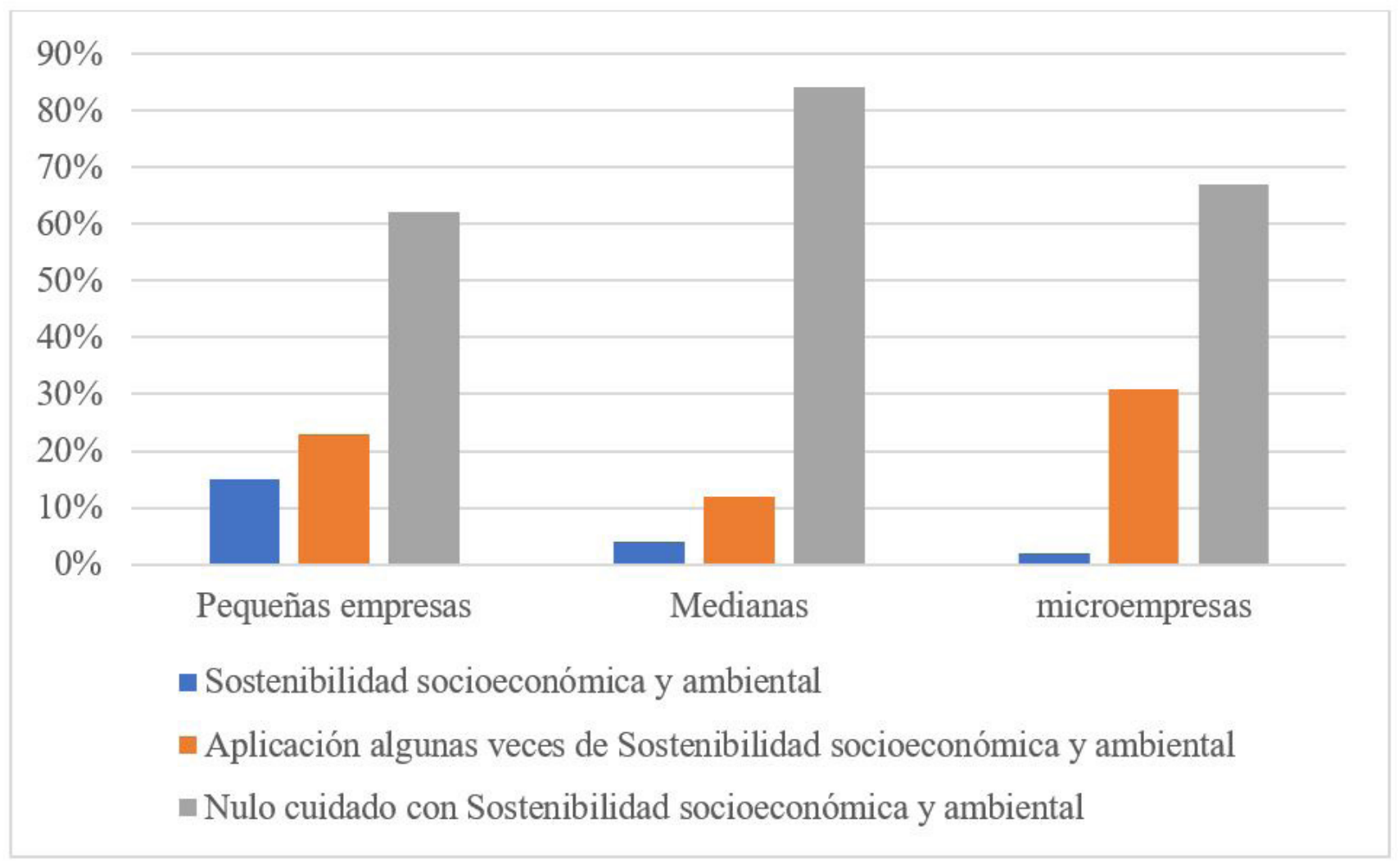

Fuente: Elaboración propia, 2019.

empresas, el 26\% de las medianas y el $8 \%$ de las microempresas, reportaron realizar siempre este tipo de capacitaciones. Si bien el 30\%, el 22\% y el 28\%, respectivamente, mencionaron hacerlo algunas veces, sigue siendo un porcentaje muy bajo, si se tiene en cuenta que no es posible alcanzar objetivos ambientales sostenibles en el tiempo cuando no se realizan capacitaciones regulares en el tema, las cuales pueden contribuir a transformar la cultura de la organización en ese sentido. 
Por último, el ejercicio del voluntariado, como un indicador de involucramiento de las empresas con las necesidades de las comunidades, en los tres casos es muy bajo para las Mipymes de la Sabana de Occidente. Tan solo el 12\% de las pequeñas empresas, el $10 \%$ de las medianas y el $4 \%$ de las microempresas reportaron acciones de voluntariado.

Con estos resultados, es posible evidenciar que las empresas encuestadas se han centrado más en los stakeholders que están cercanos al núcleo del negocio (clientes y empleados) y que aún tienen que abrirse a considerar y atender las necesidades e intereses de otros stakeholders externos, tales como los proveedores, distribuidores, acreedores y comunidades en general, para ser responsablemente competitivos desde el emprendimiento y empresarismo que se genera desde las Mipymes, el cual se puede fortalecer día a día para potencializar la economía en la provincia de Sabana de Occidente.

\section{Conclusiones}

A manera de síntesis y conclusión, los hallazgos más importantes de la investigación apuntaron a que las Mipymes requieren fortalecer los conocimientos sobre Responsabilidad Social Empresarial y el modo como los pueden integrar en la planeación estratégica, principalmente desde la misión y la visión organizacional corporativa, con el propósito de ser implementados en la práctica, a través del alcance de los objetivos empresariales con énfasis en RSE y ser más competitivos en el mercado local, provincial, regional y nacional, teniendo en cuenta que,

Para instituir una política RSE y establecer el liderazgo necesario para implementarla, una organización no solo debe realizar acciones concretas o desarrollar ciertas prácticas, sino que debe llevarla más allá, e incorporar políticas de RSE como parte de la visión y misión, y de sus prácticas diarias. (Henríquez y Oreste, 2015: 15, citado en Pérez, Espinoza y Peralta, 2016: 1).
No necesariamente los principios y valores que actualmente tienen las pequeñas, medianas y micro empresas están basados en los pilares de la RSE. Alinear ambos aspectos puede ayudarles a generar políticas y estrategias a través de las cuales dichos principios y valores de la RSE puedan ser llevados a la práctica satisfactoriamente, en pro del desarrollo socioeconómico y cultural, fortaleciendo así la competitividad en el mercado de la provincia de Sabana de Occidente. Siendo de gran importancia que los "Socios y accionistas [estén] al corriente de los principios y valores por los que se rige la entidad, por lo que la empresa debe facilitarles todo tipo de documentación" (Gómez y Martínez, 2015: 40).

Los resultados evidenciaron que la RSE aún no está integrada en los procesos de planeación de la gran mayoría de estas empresas, no sólo porque aún no se comprende el concepto de RSE en profundidad, sino porque se evidenciaron debilidades en el proceso mismo de planeación, lo cual demuestra la escasa competitividad de las empresas a nivel de la provincia de Sabana de Occidente. Así la RSE podría impulsar el desarrollo y posicionamiento de la región, y ayudar a manejar adecuadamente

La intervención de organismos de control externos a la empresa, como lo puede ser una autoridad central, tendría como consecuencia un incremento en los costos de producción de las empresas, situación que en el largo plazo puede impactar en su competitividad y estabilidad financiera (Accinelli y De la Fuente, 2013: 1).

Los procesos de planeación en estas organizaciones deben fortalecerse a través del uso de herramientas adecuadas. Entre más fuerte sea un proceso de planeación, más posibilidad hay de gestionar asertivamente las decisiones que se toman en el día a día de la operación. Las empresas con valores y principios, claros y debidamente socializados, así como una visión, una misión y una planeación organizacional que influyan significativamente en las decisiones, están mejor prepara- 
das para introducir los objetivos de la RSE en la gestión y por ende ser más competitivas en actual mercado. Lo mencionado anteriormente lleva a que

Los objetivos estratégicos de planeación de responsabilidad social alcancen una mejor y mayor participación de los actores interesados, de modo que se genere mayor lealtad, valor agregado en cuanto a productividad, calidad en la prestación de los servicios y competitividad. De ahí la importancia de que la institución revise en qué prácticas se está fallando y genere un plan de mejora (Buchelli, Yara y Africano, 2016: 1).

En cuanto a la relación con los stakeholders, se evidenció que las empresas de la Sabana de Occidente se esfuerzan por considerar y atender principalmente las necesidades e intereses de los clientes, proveedores, distribuidores y acreedores, y que incluso han desarrollado mecanismos para este propósito. Los empleados son también un stakeholder importante, pues más de la mitad de las empresas se ocupan activamente de su bienestar y desarrollo. La relación con estos grupos de interés se constituye como el campo más fértil para la introducción de objetivos de la RSE, teniendo en cuenta que es ahí donde las empresas están más preparadas y pueden comprender con mayor facilidad la relación de la RSE con los resultados del negocio. Así mismo, "La RSC transforma la fórmula básica de gobierno de la empresa, centrada en la relación bilateral entre accionistas y directivos (enfoque shareholder), en otra multilateral en la que participan todos los grupos de interés (stakeholders)" (Hoffmeister et al., 2005; Nieto, 2005; Giraldo et al., 2006, citados en Accinelli y De la Fuente, 2013:1).

El resultado obtenido sobre la prohibición y sanción de la no discriminación demuestra que, el pilar de la RSE al que se hace referencia en las relaciones laborales desde un marco de derechos, no ha sido debidamente interiorizado y requiere ser trabajado por medio de procesos formativos y decisiones políticas de directivas en las empresas.
Teniendo en cuenta que Guillén (2006: 176, citado en Camacho, 2015), afirma que:

Si bien es cierto que la falta de capacidad sancionadora de estas normas obstaculiza en la práctica su efectividad, es preciso aclarar que ellas cumplen una función que va mucho más allá de aplicar sanciones, o sea, lo que buscan en realidad es crear conciencia, que la práctica de una conducta responsable no sea producto de la existencia de un ente sancionador que va a actuar en caso de trasgresiones, sino por el contrario, que se adopten formas de comportamiento, a través de las cuales las empresas muestren ante los consumidores, los Estados y el público en general una imagen moralmente limpia y aceptable, y que se lleve a cabo de forma voluntaria (p.1).

Por otra parte, la relación con las comunidades es aún muy débil, y en general se puede decir que no son un stakeholder que se perciba como relevante para el desarrollo y la sostenibilidad social, económica y ambiental del negocio de las pequeñas, medianas y micro empresas. Por tal razón, se debe tener en cuenta que,

La apertura económica y comercial de los mercados ha planteado nuevos retos para las empresas, destacando esencialmente el de su persistencia frente a la competitividad. Esta competitividad ha implicado establecerse más allá de su estricta lógica de generación de riqueza y empleo, a efecto de reorientar su rol en agentes de cambio y desarrollo para las comunidades en las cuales se desenvuelven. (Camacho, 2015:1).

Finalmente, se evidenció una escasa promoción y capacitación en temas ambientales, lo que a su vez es un indicador de escasez de programas y esfuerzos para lograr objetivos en esta área y despertar conciencia de la importancia en los negocios para penetrar aún más en los mercados locales, provinciales, regionales y el nacional. En este sentido, la capacitación brinda un mejor nombre en el contexto competitivo de la actualidad y se evidencia que "las imágenes en busca de 
la legitimidad es posible encontrarlas con mayor presencia en los informes publicados por empresas cuyo objeto social es de alto impacto ambiental y social." (Quinche, 2017:17). Por último, se resalta que la responsabilidad social empresarial engloba las decisiones, las medidas, las actividades y las acciones éticas realizadas por cualquier empresa en el desarrollo de la actividad económica.

\section{Referencias bibliográficas}

Accinelli, E.; y De la Fuente, J. L. (2013). Responsabilidad social corporativa, actividades empresariales y desarrollo sustentable. Revista Contaduría y Administración, 58 (3), 5-298, DOI: $10.1016 /$ S0186-1042(13)71228-2

Buchelli, E. E.; Yara, Y. K.; y Africano, E. A. (2016). Caracterización de las prácticas de responsabilidad social relacionadas con gestión del talento humano en el Hospital de San José, Bogotá D.C., Colombia. Revista repertorio medicina cirugía, 25 (2), 75-140, DOI: 10.1016/j.reper.2016.03.005

Camacho, J. I. (2015). Las normas de responsabilidad social. Su dimensión en el ámbito laboral de las empresas, Revista Latinoamericana de Derecho Social, 20, 3-29, https:// doi.org/10.1016/j.rlds.2015.06.001,

Cámara de Comercio de Facatativá. (2018). Estudio Económico 2017 - Noroccidente Cundinamarqués. Recuperado de https://ccfacatativa.org.co/wp-content/uploads/2018/02/ estudio-socioeconomico-2017.pdf

Corporación Universitaria Minuto de Dios. (2018). Beneficios de la Responsabilidad Social Empresarial. Recuperado de https:// mdc.org.co/blog-consultoria-responsabilidad-social-empresarial/
Cruz, F.O. (2015). Emprendimiento e innovación social: el intraemprendimiento en la Universidad Santo Tomás, Revista Citas, 2, 98-123.

Gómez, B.; y Martínez, R. (2015), Los valores éticos en la responsabilidad social corporativa, Revista Anagramas 14 (28), 33-50.

Guerrero, R.F.; Lenise, M.; y Ojeda-Vargas, M.G. (2016). Artículo de revisión-reflexión crítica epistemológica sobre métodos mixtos en investigación de enfermería, Enfermería Universitaria, 13 (4), 246-252, doi. org/10.1016/j.reu.2016.09.001

Espinoza, C.; y Peralta, B. (2016). La responsabilidad social empresarial y su enfoque ambiental: una visión sostenible a futuro, Revista Universidad y Sociedad, 8 (3), 169-178.

Instituto Nacional de Contadores Públicos. (2016). Responsabilidad Social Empresarial, Responsabilidad Social Empresarial para PYMES. Recuperado de https://www.incp. org.co/responsabilidad-social-empresarialpara-pymes/

La Republica.net. (2016). RSE, hacia un enfoque alineado al core business. Recuperado de https://www.larepublica.net/noticia/-rsehacia-un-enfoque-alineado-al-core-business

Quinche, F. L. (2017). La cuestión ambiental en los informes de responsabilidad social empresarial y el papel de la contabilidad. un análisis crítico del discurso a los informes de responsabilidad social empresarial de las empresas generadoras de energía en Colombia en el periodo 2003-2013, (Tesis de maestría). Bogotá, Colombia: Universidad Nacional de Colombia. 